\title{
Topical Use of Quercetin-Loaded Chitosan Nanoparticles Against Ultraviolet B Radiation
}

\author{
Wenhao Nan ${ }^{1,2+}$, Li Ding ${ }^{1,2+}$, Houjie Chen ${ }^{1,2}$, Fahim U. Khan ${ }^{1,2}$, Lu Yu ${ }^{3}$, Xinbing Sui ${ }^{4,5 *}$ and \\ Xiaojun Shi ${ }^{1,2 *}$
}

${ }^{1}$ School of Life Sciences, Tsinghua University, Beijing, China, ${ }^{2}$ Graduate School at Shenzhen, Tsinghua University, Shenzhen, China, ${ }^{3}$ Shenzhen Modo Biotech Co., Ltd., Shenzhen, China, ${ }^{4}$ Department of Medical Oncology, Holistic Integrative Oncology Institutes and Holistic Integrative Cancer Center of Traditional Chinese and Western Medicine, The Affiliated Hospital of Hangzhou Normal University, College of Medicine, Hangzhou Normal University, Hangzhou, China, ${ }^{5}$ Department of Cancer Pharmacology, Holistic Integrative Pharmacy Institutes, College of Medicine, Hangzhou Normal University, Hangzhou, China

\section{OPEN ACCESS}

Edited by:

Jianxun Ding,

Changchun Institute of Applied Chemistry (CAS), China

Reviewed by:

Xiaolong $\mathrm{Li}$

Dana-Farber Cancer Institute,

United States

Tianjiao Ji,

Boston Children's Hospital and Harvard University, United States

Xinbing Sui

hzzju@zju.edu.cn

Xiaojun Shi

shixj@sz.tsinghua.edu.cn

tThese authors have contributed equally to this work.

Specialty section:

This article was submitted to Experimental Pharmacology and Drug

Discovery,

a section of the journal Frontiers in Pharmacology

Received: 15 June 2018 Accepted: 09 July 2018

Published: 26 July 2018

Citation:

Nan W, Ding L, Chen H, Khan FU, Yu L, Sui X and Shi X (2018) Topical Use of Quercetin-Loaded Chitosan Nanoparticles Against Ultraviolet B Radiation. Front. Pharmacol. 9:826. doi: 10.3389/fphar.2018.00826
Ultraviolet radiation is a major risk factor for human skin damage, especially solar ultraviolet-B (UVB) which can induce inflammation, photoaging, and skin cancer. Quercetin (Qu), one of flavonoid family members, has showed protective effects against UVB radiation. However, its application for topical use is limited by low hydrophilicity and poor percutaneous absorption. Herein, we found that Qu, if entrapped into TPPChitosan nanoparticles (TCS), can be efficiently uptake by HaCaT cells and easily permeate through the epidermis layer, meanwhile display better stability and low cytotoxicity. We also found that Qu-loaded TCs (QTCs) could notably enhance the effect of Qu on inhibiting the NF-kB/COX-2 signaling pathway as well as ameliorating the skin edema caused by UVB radiation. Therefore, this study provided a method to get rid of Qu's low hydrophilicity, enhance its percutaneous absorption and retention in the skin, and further improve its anti-UVB effect, and demonstrated that Qu-loaded chitosan nanoparticles can be used as the therapeutic agent for topical use against UVB radiation.

\section{Keywords: Quercetin, chitosan, nanoparticles, topical use, anti-UVB radiation, skin damaging}

\section{INTRODUCTION}

Previous studies have reported that the ultraviolet radiation (UVR) harms the human skin, particularly in fair skin populations (Madronich and Gruijl, 1993; Gallagher and Lee, 2006; Morganroth et al., 2013). Currently, due to gradual depletion of the ozone layer caused by chemical compounds containing gaseous chlorine or bromine from industries or human in atmosphere, the solar ultraviolet-B (UVB; $280-315 \mathrm{~nm}$ ) radiation significantly penetrates to Earth's surface resulting in skin diseases including erythema, sunburn, inflammation, photoaging, oxidative stress, DNA damage, immunosuppression, and even skin cancer (Kerr and Mcelroy, 1993; Pal et al., 2015).

One of the most important mechanisms involved in UVB radiation caused skin damage is the activation of NF- $\mathrm{kB} /$ cyclooxygenase-2 (COX-2) signaling pathway. COX-2 one of the enzyme plays a key role in converting arachidonic acid (AA) into prostaglandins (PGE2) which increases the vascular permeability and promotes edema (Kim et al., 2005; Liu et al., 2009; Hur et al., 2010; 
Pal et al., 2015; Tang et al., 2017). In addition, high COX-2 level are frequently correlated with skin inflammation and cancer development (Pentland et al., 1999; Tang et al., 2008; Mccarty, 2012; Jiao et al., 2014). Once our skin suffers from UVB radiation, IkB- $\alpha$ is phosphorylated through a family of serine/threonine kinases known as IKK, and subsequently falls off from NF- $\mathrm{B}$ protein complex, which drives NF- $\mathrm{B}$ translocating from the cytoplasm to the nucleus. Furthermore, this kind of NF- $\kappa$ B translocation enhances the expression of COX-2 (Kim et al., 2005; Liu et al., 2009; Hur et al., 2010; Pal et al., 2015; Tang et al., 2017).

Many flavonoids including Quercetin $(\mathrm{Qu})$ play a vital role in regulating NF- $\mathrm{B} / \mathrm{COX}-2$ signaling pathway (Serafini et al., 2010; de Alencar Filho et al., 2016). Qu is one representative flavonoid with anti-oxidant, anti-inflammation, and anti-tumor properties (Formica and Regelson, 1995). Previous research has revealed that $\mathrm{Qu}$ is very effective against UVB radiation through downregulating COX-2 level in vitro (Steerenberg et al., 1997). However, in vivo application of $\mathrm{Qu}$ is limited by its low hydrophilicity and poor percutaneous absorption (Hung et al., 2012). Therefore, many researchers managed to seek for new dosage forms of $\mathrm{Qu}$ to solve this problem, including Qu-loaded liposome or nanoparticles (NPs) (Hatahet et al., 2016). Our previous study has also reported using PLGA-TPGS NPs to load Qu (Zhu et al., 2016). However, although the application of PLGA-TPGS NPs significantly enhanced the protective effect of $\mathrm{Qu}$, we think it is still not ideal preparation of $\mathrm{Qu}$ considering the natural disadvantages of PLGA-TPGS NPs. That is, PLGA-TPGS NPs, owing negative surface charge, will no doubt impede the nano-bio interaction with skin cell cytomembrane which also negatively charged (Yang et al., 2009).

Chitosan (CS), an abundant linear cationic biopolymer, has several favorable biological characteristics such as biodegradability, non-toxicity, biocompatibility, and anti-pathogen (Enríquez et al., 2006). Most importantly, CS NPs (CSs) can increase the cellular uptake due to their positive surface charge which can enhance the interaction with negatively surface charged cytomembrane (Schipper et al., 1997; Huang et al., 2002). Another advantage of CSs is its enhancement of the skin permeation of hydrophobic drugs and promotion of the retention of drugs in the epidermis, due to its interaction with the skin surface which will change the morphology of the stratum corneum and break the close conjugation of the corneocyte layers (Tan et al., 2011). Thus, theoretically Qu, if loaded into CSs, may efficiently overcome the shortcomings of $\mathrm{Qu}$ for topical use to protect skin from UVB radiation damage.

In this work, we compared the protective effects of $\mathrm{Qu}$ and Qu-loaded TPP-Chitosan NPs (QTCs) against UVB radiation in vitro and in vivo, using $\mathrm{HaCaT}$ cells and C57BL/6 mice models. We found that QTCs which displayed better stability and low cytotoxicity could be uptake by HaCaT cells efficiently and easily permeate through stratum corneum and epidermis. Besides, the application of CSs carrier enhanced the effect of Qu on inhibiting the NF- $\mathrm{B} / \mathrm{COX}-2$ signaling pathway, further ameliorating the skin edema induced by UVB radiation. In this study, we firstly showed the advantages of Qu-loaded CS NPs for topical use against UVB radiation-induced skin damage.

\section{MATERIALS AND METHODS}

\section{Chemicals}

Quercetin and coumarin-6 were purchased from SigmaAldrich (St. Louis, MO, United States). Chitosan (deacetylation degree $\geq 95 \%$, viscosity 100-200 mpa.s, biotechnology level) and sodium tripolyphosphate (TPP) were purchased from Macklin (Shanghai, China). 4,6-Diamidino-2-phenylindole dihydrochloride (DAPI) and dimethyl sulfoxide (DMSO) were purchased from Sangon Biotech (Shanghai, China). Wheat Germ Agglutinin Alexa Fluor 594 conjugate (WGA-594) were purchased from Thermo Fisher Scientific (Eugene, OR, United States). All other chemicals and reagents of the highest quality were commercially available and used as received. Antibodies against p-IkB- $\alpha, \mathrm{NF}-\kappa \mathrm{B}, \mathrm{COX}-2, \mathrm{GAPDH}, \beta$-actin and lamin $\mathrm{A} / \mathrm{C}$ were purchased from Cell Signaling Technology.

\section{Formulation and Characterization of NPs}

The preparation of TPP-Chitosan NPs (TCs) was based on ionic gelation of chitosan with TPP anions reported before (Zhang et al., 2008), but with some modifications. Under magnetic stirring at room temperature, $20 \mathrm{mg}$ CS was dissolved into $10 \mathrm{ml}$ $1 \%(\mathrm{~V} / \mathrm{V})$ acetum and adjusted the $\mathrm{pH}$ to 5.5 , then $3.5 \mathrm{ml}$ TPP with the concentration of $2 \mathrm{mg} / \mathrm{ml}$ was dropwise added into CS solution. Opalescent and transparent solution was formed after transient sonication. Qu loaded TPP-Chitosan NPs (QTCs) were formed by dropwise adding $1 \mathrm{ml}$ Qu ethanol solution with the concentration of $3 \mathrm{mg} / \mathrm{ml}$ into CS solution before adding TPP. The NPs were centrifuged at $8,000 \mathrm{rpm}, 4^{\circ} \mathrm{C}$ for $30 \mathrm{~min}$, the supernatant was collected for HPLC and the precipitation was suspended after washed three times by $\mathrm{ddH}_{2} \mathrm{O}$, and finally lyophilized.

The hydrodynamic diameter and zeta potential of NPs were measured by Dynamic Light Scattering Zetasizer (Zetasizer Nano ZS90, Malvern Instruments Ltd., Malvern, United Kingdom). The morphology of NPs was showed by transmission electron microscopy (TEM, Tecnai G20, FEI Company, Hillsboro, OR, United States).

\section{Measurement of Drug Encapsulation Efficiency and Drug Loading Content}

The drug encapsulation efficiency (EE) and drug loading content (LC) of the QTCs were measured by HPLC (LC 1200, Agilent Technologies, Santa Clara, CA, United States). The mobile phase of HPLC was $0.2 \%$ phosphoric acid solution and $\mathrm{MeOH}$ with the volume ratio of 55:45. The flow rate of mobile phase was $1 \mathrm{ml} / \mathrm{min}$. The UV detection wavelength was $360 \mathrm{~nm}$. A C-18 column $(150 \mathrm{~mm} \times 4.6 \mathrm{~mm}$, GL Science Inc., Tokyo, Japan) was used. The supernatant of QTCs after centrifugation was used for HPLC to detect the concentration of $\mathrm{Qu}$ in the supernatant; therefore, we can detect the amount of $\mathrm{Qu}$ in supernatant $\left(\mathrm{W}_{1}\right)$. We named the weight of lyophilized precipitation as 
$\mathrm{W}_{2}$ and the amount of $\mathrm{Qu}$ added in the preparation of QTC as $\mathrm{W}_{3}$.

$$
\begin{aligned}
& \mathrm{EE}=\left(\mathrm{W}_{3}-\mathrm{W}_{1}\right) / \mathrm{W}_{3} \times 100 \% \\
& \mathrm{LC}=\left(\mathrm{W}_{3}-\mathrm{W}_{1}\right) / \mathrm{W}_{2} \times 100 \%
\end{aligned}
$$

\section{Cell Culture}

HaCaT cells were cultured in Dulbecco's modified Eagle's medium (DMEM; Gibco BRL, United States) supplemented with $10 \%$ fetal bovine serum (FBS, Gibco) at $37^{\circ} \mathrm{C}$ with $5 \% \mathrm{CO}_{2}$.

\section{Cellular Uptake of Coumarin-6-Loaded TCs and Its Efficiency}

Coumarin-6 was used as the fluorescent probe which was entrapped into TCs to show the cellular uptake of QTCs. HaCaT cells were seeded in 12-well plate containing cover glasses in the bottom. After the cells adhered to the glasses, $100 \mu \mathrm{g} / \mathrm{ml}$ coumarin-6-loaded TCs was added to the culture medium and incubated at $37^{\circ} \mathrm{C}$ for $3 \mathrm{~h}$. Then removed the culture medium and washed with PBS three times. Next added WGA-594 diluted by PBS to the wells and incubated at $37^{\circ} \mathrm{C}$ for $20 \mathrm{~min}$ to stain the cytomembrane, then washed with PBS three times and fixed with $4 \%$ paraformaldehyde for $20 \mathrm{~min}$, finally, cells were stained with DAPI for 15 min and washed with PBS three times. Thereafter, cells were investigated by confocal laser scanning microscope (CLSM, Olympus Fluoview FV-1000, Tokyo, Japan).

To quantitatively measure the cellular uptake efficiency of coumarin-6-loaded TCs, $100 \mu \mathrm{g} / \mathrm{ml}$ coumarin-6-loaded TCs were incubated with $\mathrm{HaCaT}$ cells in 12-well plate at 3, 6, 12, 24 (each group, $n=3$ ), and the quantitative ratio of coumarin- 6 fluorescence in cytoplasm were measured by detecting the absorbance value at $320 \mathrm{~nm}$.

\section{Cytotoxicity and UVB Protective Experiment in Vitro}

Cytotoxicity was evaluated by MTT assay. Cells were seeded in 96-well plate, after the cell attachment rate reached $60 \sim 70 \%$, replaced the culture medium to new culture medium mixed with or without different concentrations of $\mathrm{Qu}$ (mixed with DMSO, at the concentration of no influence on cells), QTCs, TCs and incubated for another $24 \mathrm{~h}$. Then removed the culture medium, added new culture medium mixed with MTT $(0.5 \mathrm{mg} / \mathrm{ml})$ and incubated $4 \mathrm{~h}$. Finally, the absorbance value of $490 \mathrm{~nm}$ was detected.

The method to investigate the UVB protective effects of $\mathrm{Qu}$, QTCs, and TCs in vitro were almost the same as cytotoxicity experiment. The difference was the time of incubation with different concentrations of $\mathrm{Qu}, \mathrm{QTCs}$, and TCs been cut to $8 \mathrm{~h}$, then removed the culture medium and irradiated using a microprocessor-controlled UV Crosslinker (XL-1000, SPECTROLINKER ${ }^{\mathrm{TM}}$, United States) of $12 \mathrm{~mJ} / \mathrm{cm}^{2}$. After the radiation, cells were added new drug free culture medium and returned to the incubator immediately.

\section{Immunoblotting}

Cell and tissue lysates were separated by 12\% SDS-PAGE and analyzed by immunoblotting using P-IkB- $\alpha$, NF- $\kappa$ B, COX-2 antibodies, followed by enhanced chemiluminescence (ECL) detection. Isolation of nuclear protein was conducted using the manufacturer's protocol (Abcam, Cambridge, MA, United States).

\section{Immunocytochemical Staining of NF-кB}

Cell culture and UVB radiation were the same as the UVB radiation treatment mentioned before. $10 \mathrm{~h}$ after UVB radiation, the cells were fixed and immunofluorescence stained with NF- $\mathrm{\kappa B}$ antibody. After stained with DAPI, the cells were detected by CLSM.

\section{Percutaneous Absorption and Retention Study of QTCs and Qu Aqueous Solution}

All animal experiment protocols were approved by the Administrative Committee on Animal Research in the Graduate School at Shenzhen, Tsinghua University. Female C57BL/6 mice (aged 6 weeks, weighing about $20 \mathrm{~g}$ ) were purchased from Guangdong Medical Laboratory Animal Center. After execution, the dorsal skin was carefully shaved with electric clippers and then carefully cut down the dorsal skin. Scrape the subcutaneous tissue completely and carefully by using a knife, and then fixed the skin on the V-C diffusion cell to investigate the percutaneous absorption of QTCs aqueous solution and Qu aqueous solution (each group, $n=3$ ). Take samples regularly on $2,4,6,8,12$, and $24 \mathrm{~h}$ for HPLC. The preparation of QTCs aqueous solution was mentioned before, and the preparation of $\mathrm{Qu}$ aqueous solution was by dropwise adding $1 \mathrm{ml}$ Qu ethanol solution $(5 \mathrm{mg} / \mathrm{ml})$ to

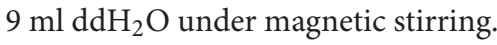

\section{Skin Permeation of QTCs and Its Mechanism}

Coumarin-6-loaded TCs was applied to represent skin permeation of QTCs visually. After the mice dorsal skin was carefully shaved with electric clippers, Coumarin-6-loaded TCs aqueous solution $(80 \mathrm{mg} / 20 \mathrm{ml})$ was applied every $3 \mathrm{~h}$, and after $12 \mathrm{~h}$ the treated skin was excised for frozen section to observe the skin permeation of Coumarin-6-loaded TCs. Divided the dorsal skin shaved mice into three groups (one group was for control with no treatment) and used the same method to treat shaved dorsal skin by $\mathrm{H}_{2} \mathrm{O}$ and QTCs aqueous solution, then the treated skin was excised and the paraffin section was processed for hematoxylin and eosin (HE) staining to observe the difference of stratum corneum between different treated skins.

\section{UVB Radiation on Animal, Staining, and Histopathological Analysis}

After anesthesia with chloral hydrate, the dorsal skin of C57BL/6 mice was carefully shaved with electric clippers, depilated with depilatory paste $\left(\right.$ Veet $\left.^{\circledR}\right)$ and divided into five groups (one group was for control), then $\mathrm{ddH}_{2} \mathrm{O}, 10 \mathrm{mg} / 20 \mathrm{ml}$ Qu aqueous solution and $80 \mathrm{mg} / 20 \mathrm{ml}$ QTCs and TCs aqueous solution were applied 
to the dorsal skin every $8 \mathrm{~h}$. Three days later, UVB radiation was applied at the intensity of $400 \mathrm{~mJ} / \mathrm{cm}^{2}$. After radiation, the four kinds of aqueous solutions were applied every $8 \mathrm{~h}$. After $48 \mathrm{~h}$, the mice were executed. The aqueous solution treated areas were excised, cut into small pieces, part of which were prepared paraffin sections and protein extraction. The protein extraction was used for immunoblotting, and the paraffin sections were used for HE and Masson staining which were further used for histopathological analysis. The other part of treated skin pieces was used for detecting the content of PGE2. Respectively weigh $100 \mathrm{mg}$ different treated skin pieces, added into $1 \mathrm{ml} \mathrm{PBS}(\mathrm{pH}$ 7.2) after cut into small pieces with intermittent ultrasonic for $1 \mathrm{~h}$ and then detected the absorbance value at $278 \mathrm{~nm}$, Using the absorbance value per gram of skin $(\mathrm{A} / \mathrm{g})$ to represent the content of PGE2.

\section{Statistical Analysis}

All data are presented as the mean \pm SEM of no less than three independent experiments. Comparisons were performed using a two-tailed paired Student's test $\left({ }^{*} P<0.05\right.$, ${ }^{* *} P<0.01$, $\left.{ }^{* * *} P<0.001,{ }^{* * * *} P<0.0001\right)$.

\section{RESULTS}

\section{Preparation and Characterization of NPs}

We prepared the QTCs using the published protocol, but with minor modification (Zhang et al., 2008). As we presented in Figures $1 \mathrm{~A}-\mathrm{C}$, Qu can firstly adhere to CS based on similarityintermiscibility theory, and then formed nanospheres through TPP-CS crosslinking under continuously stirring condition.

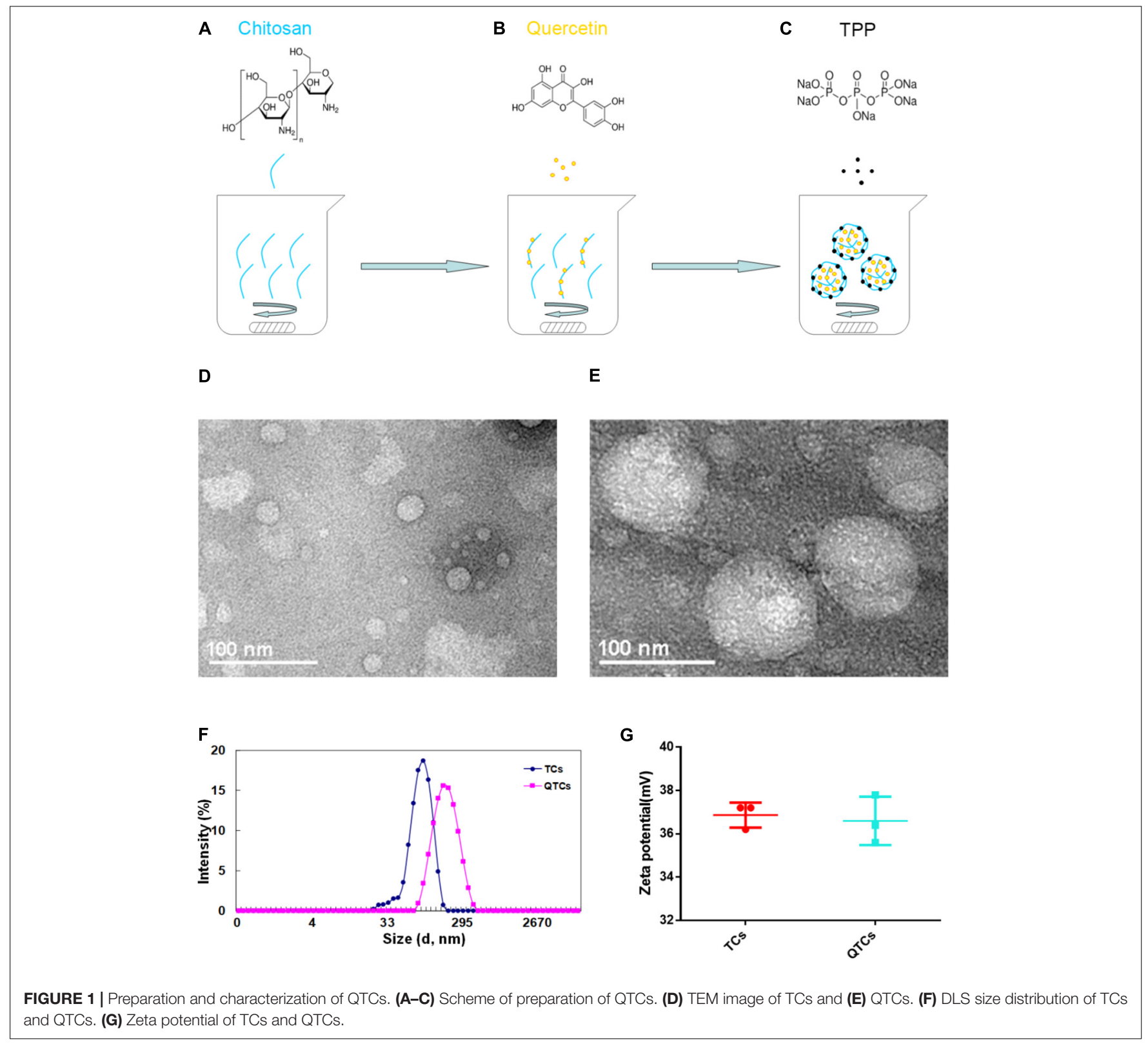


Next, the morphologies of QTCs and TCs were observed. From the TEM image, QTCs and TCs were all nearly spherical in shape, but the size between each was quite different (Figures 1D,E). The dynamic light scattering (DLS) assay showed that the average size of TCs was about $89.48 \pm 2.03 \mathrm{~nm}$ while QTCs was about $183.63 \pm 1.52 \mathrm{~nm}$ in Figure 1F, consistent with the results of TEM. Moreover, zeta potential, an essential index in the stability of NPs in suspension through electrostatic repulsion between the NPs, had been tested. As the result presented in Figure 1G, both QTCs and TCs showed almost the same zeta potential, about $37 \mathrm{mV}$ in average. This data implied that both QTCs and TCs were highly stable in suspension. Then the drug EE and LC of QTCs were measured by HPLC. EE and LC of QTCs were $90.98 \pm 1.66 \%$ and $13.15 \pm 0.77 \%$, respectively, indicating the excellent drug carrier ability of QTCs.

\section{Cellular Uptake, Cytotoxicity, and UVB Protective Effect of QTCs in Vitro}

Many studies have been reported that CSs could enhance cellular uptake because their positive surface charge can promote the bio-nano interaction with the cytoplasmic-membrane which has negative surface charge (Schipper et al., 1997; Huang et al., 2002), which is also the reason why CSs have been often used as gene delivery carrier (Özbas̨-Turan and Akbuğa, 2011). In our study, the enhancement UVB protective effect of QTCs depends on the successful internalization and sustained retention by skin cells. Therefore, we used coumarin- 6 which is a kind of fluorescent dye, instead of $\mathrm{Qu}$ in order to visually investigate the cellular uptake of QTCs and its efficiency. HaCaT cells, the most commonly used human skin cells, were chosen as representative cells. Figure $\mathbf{2 A}$ showed the confocal images of HaCaT cells after incubation with coumarin6-loaded TCs for $3 \mathrm{~h}$. The data suggested coumarin-6-loaded TCs can be ingested by $\mathrm{HaCaT}$ cells and internalized into the cytoplasm. Moreover, to investigate the cellular uptake efficiency of QTCs, we measured the quantitative ratio of coumarin-6 fluorescence in cytoplasm at indicated times after incubation of coumarin-6-loaded TCs with HaCaT cells. The result showed that $\mathrm{HaCaT}$ cells could almost consistently uptake coumarin-6-loaded TCs in $24 \mathrm{~h}$. The cellular uptake efficiency was gradually increased and reached at about 50\% at $24 \mathrm{~h}$ (Figure 2B).

Next, we evaluated the cytotoxicity of Qu and QTCs in $\mathrm{HaCaT}$ cells. Figure $\mathbf{2 C}$ showed the in vitro cell viability of the drug formulated in QTCs and Qu at equivalent concentrations of 5,10,20,40 $\mathrm{g} / \mathrm{ml}$, respectively. High dose free $\mathrm{Qu}$ showed obvious cytotoxicity compared with QTCs at the same equivalent concentration at $24 \mathrm{~h}$, which indicated that QTCs can significantly reduce cytotoxicity of high dose Qu. Additionally, the TCs did not show any obvious toxicity against HaCaT cells. After verifying the safety of QTCs, we furthermore tried to investigate the protective effect of QTCs compared to $\mathrm{Qu}$ on $\mathrm{HaCaT}$ cells under UVB radiation.
A
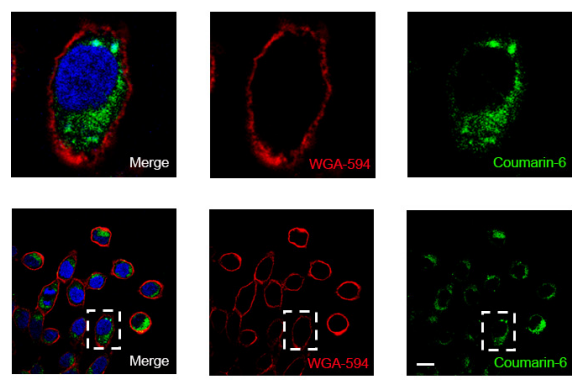

C

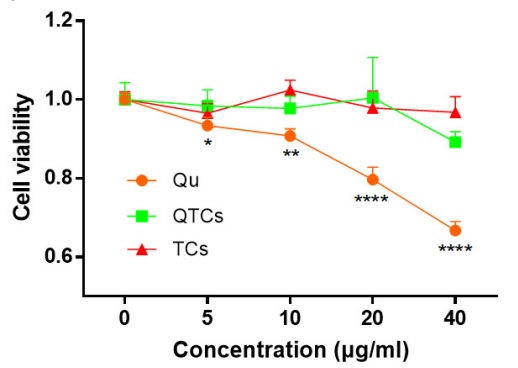

B

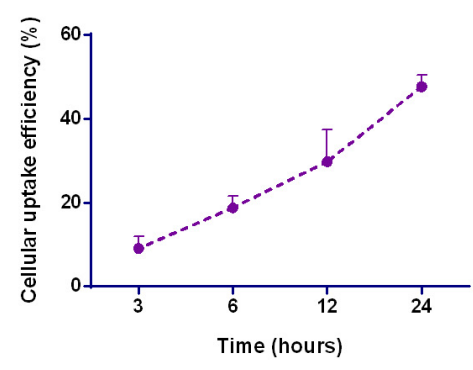

D

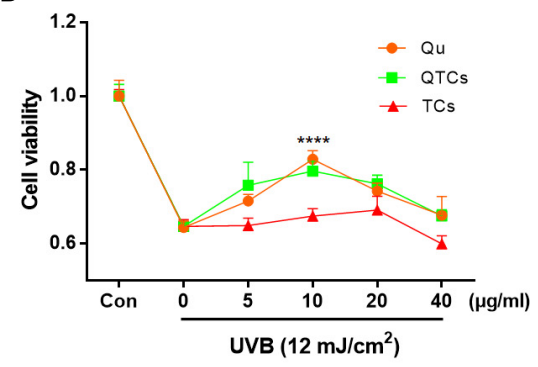

FIGURE 2 | Cellular uptake, cytotoxicity, and UVB protective effect of QTCs in vitro. (A) Cellular uptake of coumarin-6-loaded TCs (100 $\mu$ g/ml) was tested by CLSM after $3 \mathrm{~h}$ incubation with HaCaT cells. The images above are the enlarged ones in the white box on the below images. Cytomembrane was labeled with WGA-594 (red). Scale bar: $10 \mu \mathrm{m}$. (B) The cellular uptake efficiency of coumarin-6-loaded TCs (100 $\mu \mathrm{g} / \mathrm{ml})$ was measured at indicated times. (C) Cytotoxicity evaluation of Qu, QTCs, and TCs after $24 \mathrm{~h}$ incubation with HaCaT cells. (D) UVB protective effect evaluation of Qu, QTCs, and TCs. HaCaT cells were firstly incubated with Qu, QTCs or TCs, respectively, for $8 \mathrm{~h}$ and then treated with $12 \mathrm{~mJ} / \mathrm{cm}^{2}$ UVB radiation. Concentration of QTCs was at the same drug dose compared to Qu, and that the TCs was at the same nanoparticles concentration with QTCs $\left({ }^{*} P<0.05,{ }^{* *} P<0.01\right.$, $\left.{ }^{* * * *} P<0.0001\right)$. 
A

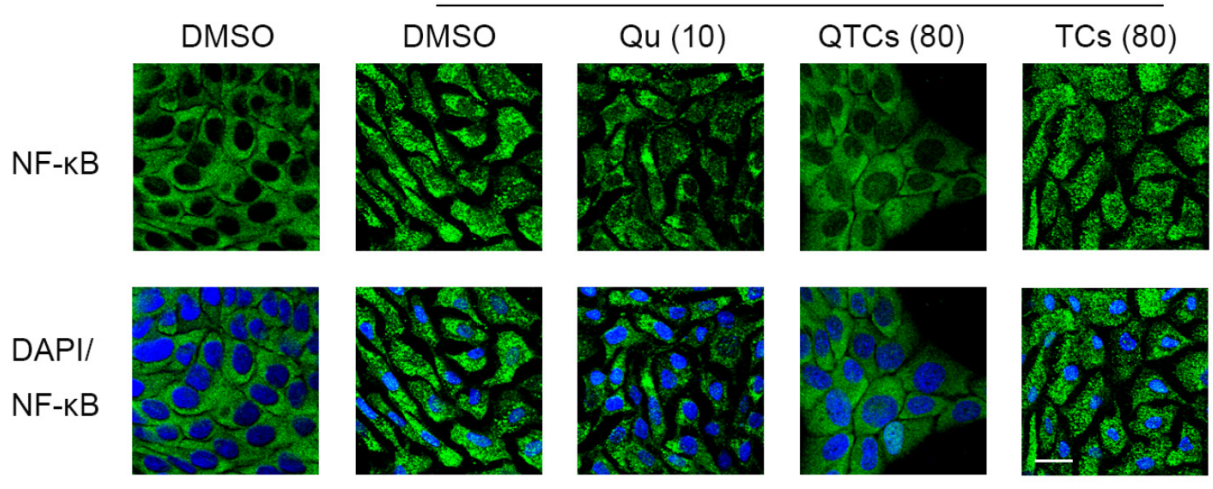

B
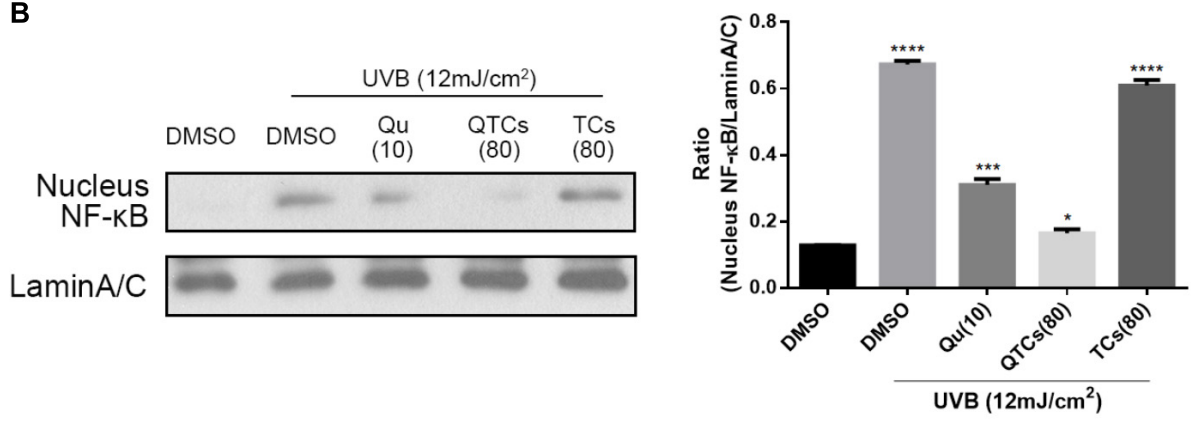

C
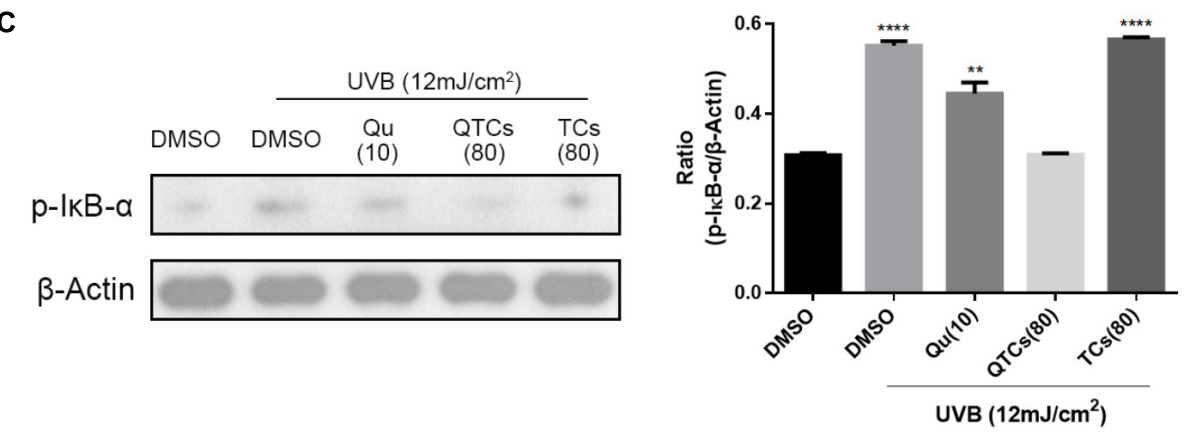

D

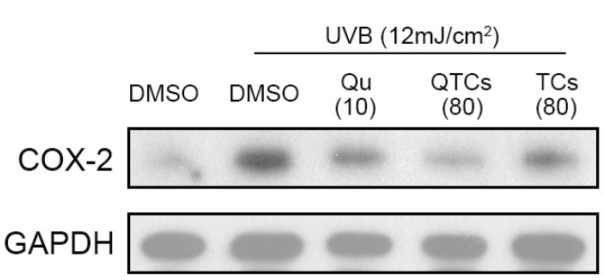

FIGURE 3 | QTCs enhance the inhibition effect of Qu on the NF-kB/COX-2 signaling pathway in vitro. (A) Effect of Qu, QTCs, and TCs on the translocation of NF-kB from cytoplasm to the nuclei was visually illustrated by CLSM. Scale bar: $20 \mu \mathrm{m}$. (B) Immunoblotting analysis of the effect of Qu, QTCs, and TCs on the expression of NF-kB in nucleus. (C) Immunoblotting analysis of the effect of Qu, QTCs, and TCs on the phosphorylation of IkB- $\alpha$. (D) Immunoblotting analysis of the effect of Qu, QTCs, and TCs on the expression of COX-2. Histograms represent quantitative analysis of the immunoblotting results $\left({ }^{*} P<0.05,{ }^{* *} P<0.01,{ }^{* * *} P<0.001\right.$, **** $P<0.0001)$.

We found that QTCs had same protective effects on HaCaT cells as Qu after $12 \mathrm{~mJ} / \mathrm{cm}^{2}$ UVB radiation (Figure 2D). At the equivalent concentration of $10 \mu \mathrm{g} / \mathrm{ml}$ of $\mathrm{Qu}$, both QTCs and
Qu showed the highest protective efficiency on UVB radiation. Meanwhile, TCs showed no significant protective effect on UVB radiation. 
A

Omin

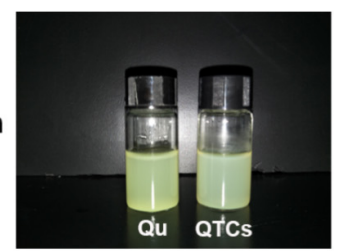

$30 \mathrm{~min}$

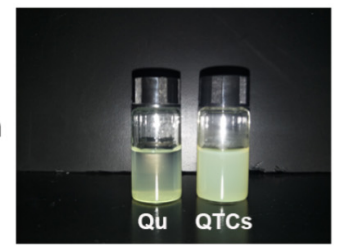

B

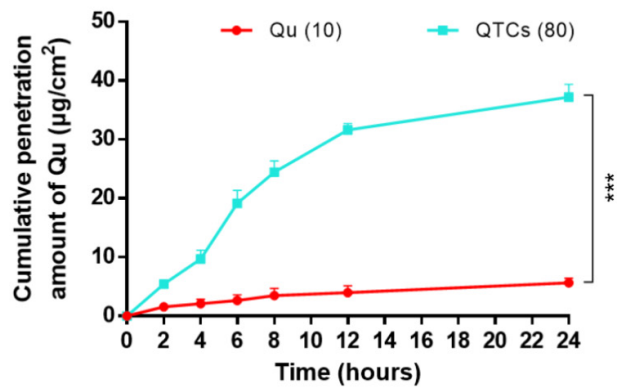

D

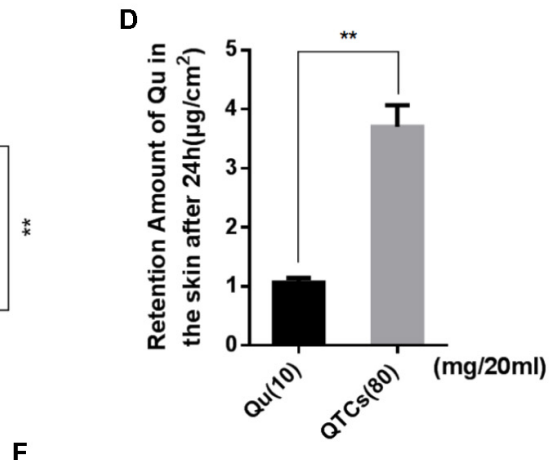

F
E

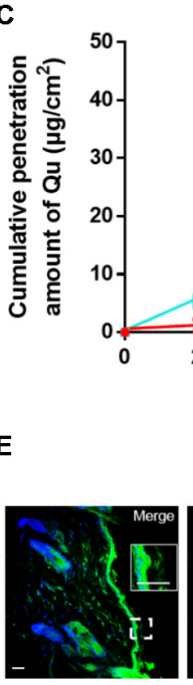

$\rightarrow$ Qu (10)

$-\operatorname{QTCs}(80)$

$Y=2.758 X+0.3444$ $2.758 X+0.3444$
$r=0.9732$

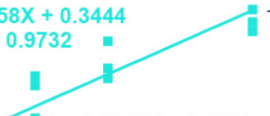

$\mathrm{H}=0.3167 \mathrm{X}+0.6080$

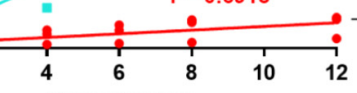

Time (hours)
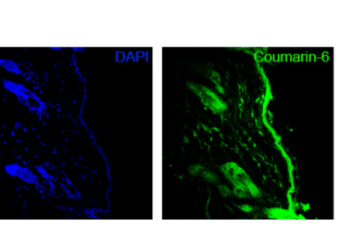
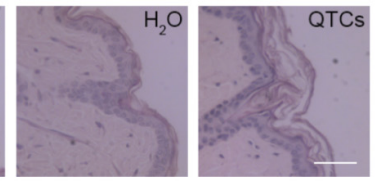

FIGURE 4 | QTCs enhance the percutaneous absorption of Qu and its mechanism. (A) Comparison of stability between Qu aqueous solution and QTCs aqueous solution. (B) Cumulative penetration amount curves of Qu and QTCs in 24 h. (C) Cumulative penetration amount curves of Qu and QTCs in 12 h. (D) Retention amount of Qu in the skin after 24 h. $\left({ }^{* *} P<0.01,{ }^{* * *} P<0.001\right)$. (E) Coumarin-6-loaded TCs permeated and deposited in mice skin. The image in the white box is enlarged vision of the small white box below. (F) HE staining of normal, $\mathrm{H}_{2} \mathrm{O}$ and QTCs aqueous solution treated mice skin. Scale bars: $50 \mathrm{\mu m}$.

\section{QTCs Enhance the Inhibition Efficacy of Qu on the NF-кB/COX-2 Signaling Pathway in Vitro}

Many pharmacological researches have revealed that $\mathrm{Qu}$ is very effective against UVB radiation via downregulating COX-2 level in vitro through $\mathrm{NF}-\kappa \mathrm{B} / \mathrm{COX}-2$ signaling pathway $(13,14)$. In non-stimulated cells, the NF- $\kappa \mathrm{B}$ dimer binds to one of the three inhibitors $(\mathrm{IkB} \alpha, \mathrm{IkB} \beta$, and $\mathrm{IkB} \varepsilon)$ and exists in an inactive state. Various signals activate NF- $\kappa$ B by degrading one or more of these three inhibitors (DiDonato et al., 1997). Therefore, the hallmark of NF- $\kappa \mathrm{B}$ activation is that the activated NF- $\kappa \mathrm{B}$ protein will enter the nucleus, and then binds to DNA in the nucleus to upregulate COX-2 level. In order to further evaluate the protective effect of QTCs, we observed immunofluorescent staining of NF- $\kappa \mathrm{B}$ protein in $\mathrm{HaCaT}$ cells with UVB radiation in different treatment groups. As can be seen in Figure 3A, after $12 \mathrm{~mJ} / \mathrm{cm}^{2} \mathrm{UVB}$ radiation, NF- $\kappa \mathrm{B}$ protein was translocated from the cytoplasm to the nucleus. But when pre-treatment with $10 \mu \mathrm{g} / \mathrm{ml} \mathrm{Qu}$, the NF- $\kappa \mathrm{B}$ protein in the nucleus reduced. And the pre-treatment $80 \mu \mathrm{g} / \mathrm{ml}$ QTCs (consistent drug dose with $10 \mu \mathrm{g} / \mathrm{ml} \mathrm{Qu}$ ) showed more reduction compared with free $\mathrm{Qu}$. TCs showed no obvious influence on this process. What must be mentioned was that the nucleus size of UVB irradiated HaCaT cells was smaller compared to normal HaCaT cells, and the morphology of UVB irradiated cells also changed to be abnormal, whereas the HaCaT cells pretreated with QTCs looked nearly normal condition after UVB irradiation. Representative western blotting image of nucleus NF- $\kappa$ B protein level also showed the same conclusion (Figure 3B). Furthermore, pre-treatment with $10 \mu \mathrm{g} / \mathrm{ml} \mathrm{Qu}$ or $80 \mu \mathrm{g} / \mathrm{ml}$ QTCs (consistent drug dose with $10 \mu \mathrm{g} / \mathrm{ml} \mathrm{Qu}$ ) can also inhibit the over-phosphorylation of IkB- $\alpha$ caused by UVB radiation (Figure $3 \mathrm{C}$ ). As a result, COX-2 protein expression level stayed relatively low in Qu or QTCs pre-treatment group (Figure 3D). Similarly, the effect of QTCs on these proteins was much stronger than free $\mathrm{Qu}$. All of these data has proved that QTCs not only just remains the Qu-like inhibition effect on the 
A
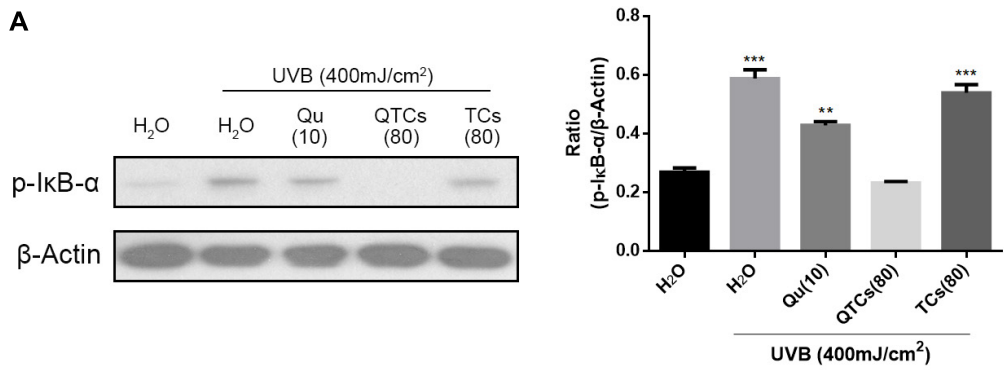

B

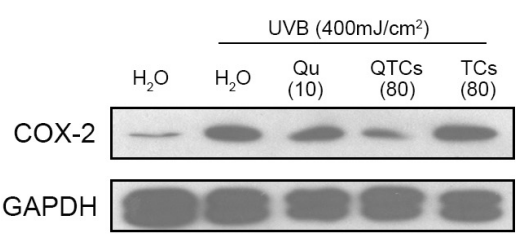

$E$

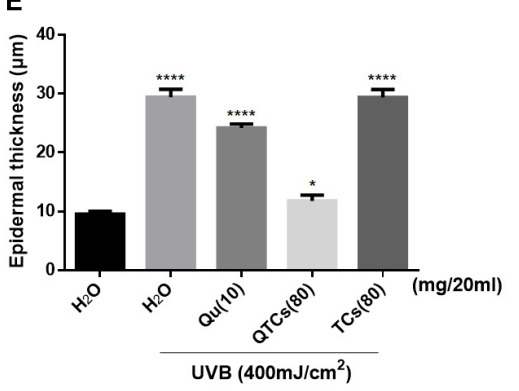

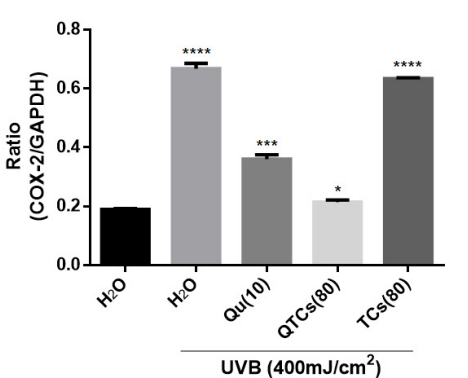

$\mathbf{F}$

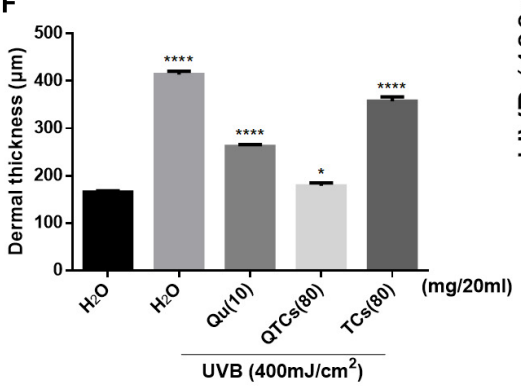

C

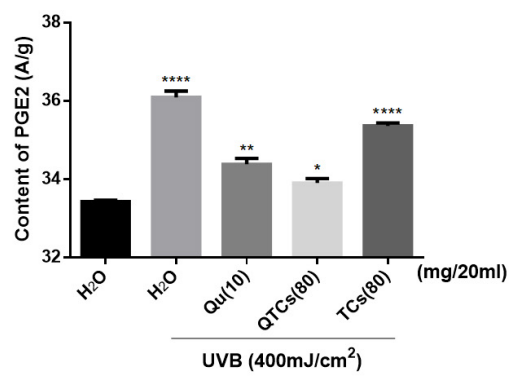

D
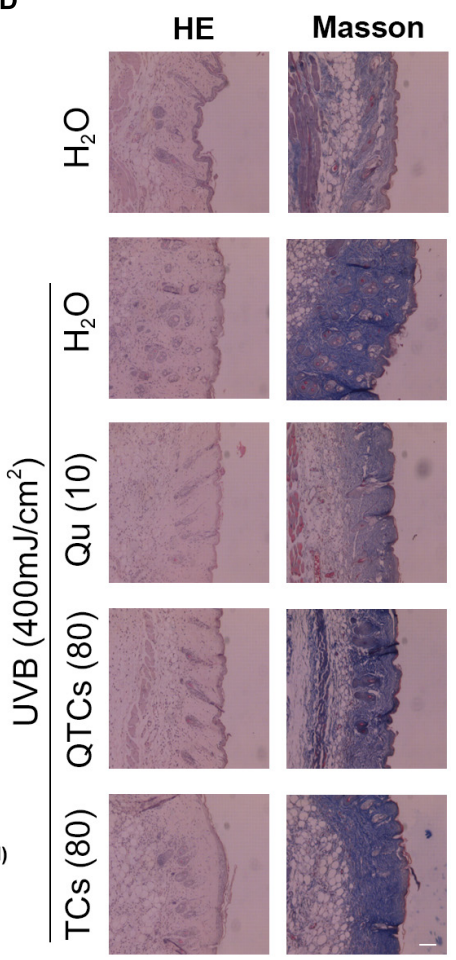

FIGURE 5 | QTCs enhance the protective effect of Qu on UVB induced skin damages in vivo. (A) Immunoblotting analysis of the effect of Qu, QTCs, and TCs on the phosphorylation of IkB- $\alpha$ and (B) COX-2. Histograms represent quantitative analysis of the immunoblotting results. (C) The content of PGE2 in the mice skin under different treatments. (D) HE and Masson staining represents the alteration of epidermis and dermis, especially the thickness. Scale bar: $50 \mu \mathrm{m}$. (E) The epidermal thickness of differently treated mice. (F) The dermal thickness of differently treated mice. $\left({ }^{*} P<0.05,{ }^{* *} P<0.01,{ }^{* * *} P<0.001,{ }^{* * * *} P<0.0001\right)$.

NF- $\kappa$ B/COX-2 signaling pathway in vitro, but also enhances its therapeutic efficacy.

\section{QTCs Enhance the Percutaneous Absorption and Retention of $\mathrm{Qu}$}

The efficiency of an external dosage form mostly depends on the percutaneous absorption. As we mentioned before, CSs is reported as nanomaterials with the advantages of enhancement of the skin permeation of hydrophobic drugs and promotion of the retention of drugs in the epidermis, due to its interaction with the skin surface will change the morphology of the stratum corneum and break the close conjugation of the corneocyte layers (Tan et al., 2011). Therefore, we next checked the contribution of CSs on percutaneous absorption and retention of $\mathrm{Qu}$ in vitro.
Firstly, we conducted the percutaneous absorption experiment of QTCs and Qu and compared the efficiency of percutaneous absorption in different groups. The results shown in Figure 4A indicated that $\mathrm{Qu}$ aqueous solution was very unstable and would precipitate within $30 \mathrm{~min}$ because of the low hydrophilicity of $\mathrm{Qu}$. And the cumulative penetration amount of $\mathrm{Qu}$ in aqueous solution was very small even after $24 \mathrm{~h}$ (Figure 4B). On the contrary, QTCs showed better stability in aqueous solution (Figure 4A) and the cumulative penetration amount of $\mathrm{Qu}$ in QTCs shown higher level after $24 \mathrm{~h}$ (Figure 4B). Moreover, the cumulative penetration amount of Qu in QTCs presented an upward tendency in a linear line within $12 \mathrm{~h}$, as showed in Figure 4C the $r$-value was 0.9732 which approaches to 1 , demonstrating that QTCs can significantly improve the percutaneous absorption efficiency of $\mathrm{Qu}$ in vitro. Further result 


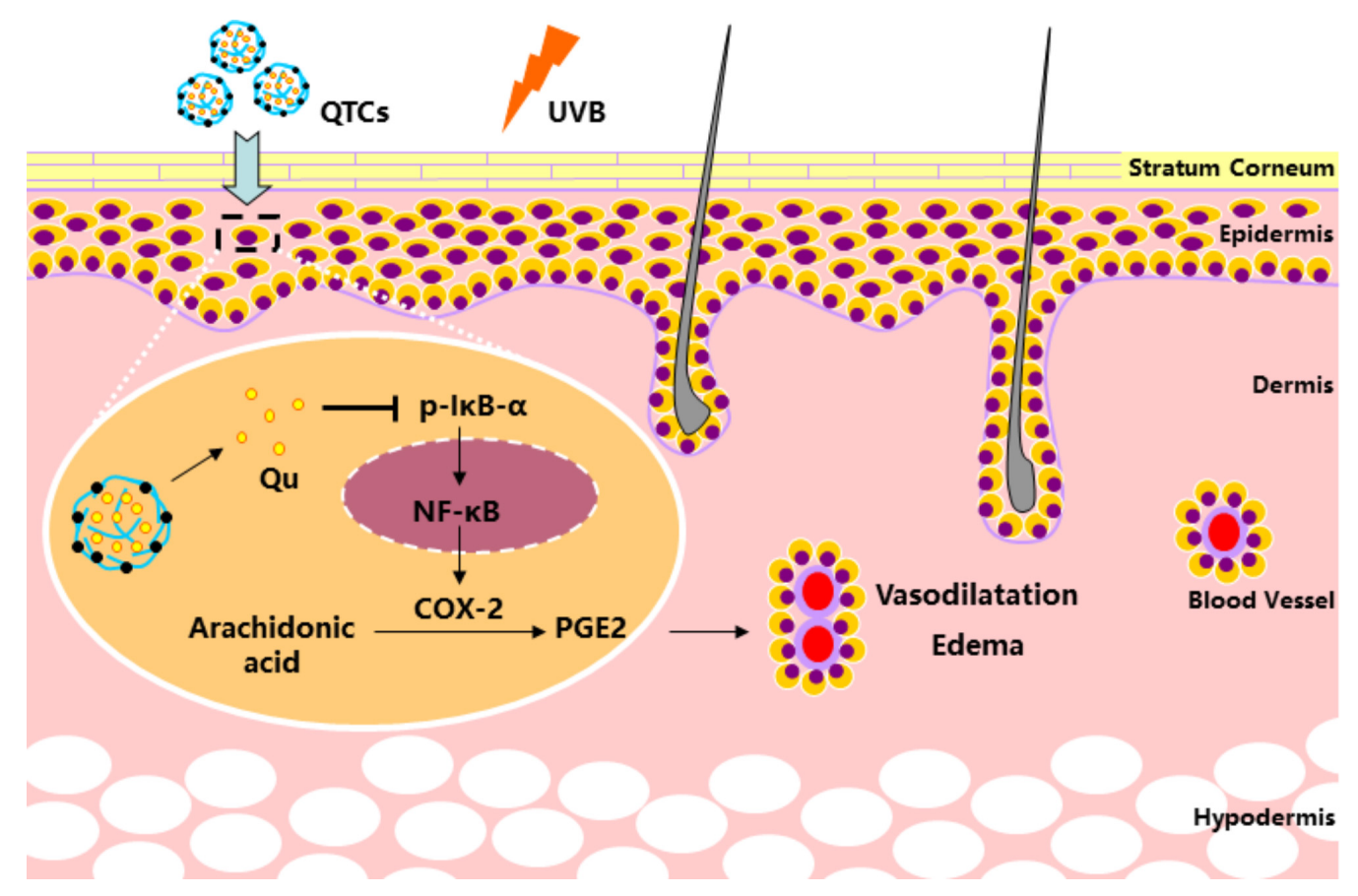

FIGURE 6 | Mechanism of QTCs on enhancing the protective effect of Qu on UVB induced skin vasodilatation and edema.

shown QTCs helped the retention of Qu in skin after $24 \mathrm{~h}$ (Figure 4D).

Then, to visually show the skin permeation of QTCs in vivo, we also used coumarin-6-loaded TCs to represent QTCs. As showed in Figure 4E, after application of coumarin-6-loaded TCs aqueous solution on the shaved mice skin after $12 \mathrm{~h}$, we found the coumarin-6-loaded TCs permeated and stored in the skin. Furthermore, we compared the HE staining of normal, $\mathrm{ddH}_{2} \mathrm{O}$ treated and QTCs aqueous solution treated mice skin, and the results showed in Figure 4F. The stratum corneum of QTCs aqueous solution treated mice skin was more swelling and had larger pore space than normal and $\mathrm{ddH}_{2} \mathrm{O}$ treated skin. This result was consistent with the enlarged vision in the white box in Figure 4E, which also showed that the stratum corneum of coumarin-6-loaded TCs aqueous solution treated skin was swelling. These results proved that QTCs can also significantly improve the percutaneous absorption efficiency of $\mathrm{Qu}$ in vivo.

\section{In Vivo Study of the Protective Effects of QTCs on UVB-Induced Skin Damage}

We further investigated the protective effects of QTCs in vivo after UVB radiation. We compared the effects of four different aqueous solution containing $\mathrm{ddH}_{2} \mathrm{O}, 10 \mathrm{mg} / 20 \mathrm{ml}$ $\mathrm{Qu}, 80 \mathrm{mg} / 20 \mathrm{ml}$ QTCs, and $80 \mathrm{mg} / 20 \mathrm{ml} \mathrm{TCs}$ in mice after UVB radiation. The immunoblotting results were shown in Figures 5A,B. From the results, it was obvious that QTCs had a significant enhancement effect on inhibiting NF- $\mathrm{B} / \mathrm{COX}-2$ signaling pathway by downregulating the phosphorylation of IkB- $\alpha$ and the expression of COX-2 when compared with
Qu. However, TCs had no notably influence on NF- $\mathrm{B} / \mathrm{COX}-2$ signaling pathway after UVB radiation.

Moreover, PGE2 can be significantly increased after UVB radiation due to the activation of $\mathrm{NF}-\mathrm{kB} / \mathrm{COX}-2$ signaling pathway, which can induce the increase of the vascular permeability and promotes skin edema (Kim et al., 2005; Liu et al., 2009; Hur et al., 2010; Pal et al., 2015; Tang et al., 2017). Herein, we quantificationally compared the content of PGE2 in different treated skins. Mice skin treated with QTCs showed less amount of PGE2 (Figure 5C). And the HE and Masson staining results in Figure 5D, which could help to intuitively see the alteration of mice skin after UVB radiation, also suggested the slighter disorder of collagen fiber and edema of the epidermis and dermis in mice skin treated with QTCs. The thickness of epidermis and dermis of mice also varied from different treatment. Consistent with the previous results, the status of QTCs-treated mice was most close to the normal mice skin (Figures 5E,F). However, TCs also had no significant difference compared with non-external preparation treatment group. With all these results, we can conclude that QTCs significantly enhanced the effect of Qu on the inhibition of the increase of PGE2 and inhibition of the edema of skin, which was closer to the normal skin, compared to $\mathrm{Qu}$ and TCs.

\section{DISCUSSION}

The pH of skin surface ranges from 5.4 to 5.9 (Eberleinkönig et al., 2000; Ali and Yosipovitch, 2013). Interestingly, the surface charge of CSs is positive in this $\mathrm{pH}$, which could increase the interaction between CSs and negatively charged stratum corneum. 
The interaction between CSs and stratum corneum changes the morphology of the stratum corneum and breaks the close conjugation of the corneocyte layers, which means CSs could notably enhance the skin permeation of hydrophobic drugs (Tan et al., 2011). What's more, due to the large quantities of amino groups on its chains, CSs exhibits a $\mathrm{pH}$-sensitive behavior, so CSs can undergo volume phase transitions from swollen to collapsed states, which can significantly change the drug release capacity (Seda Tiğli Aydın and Pulat, 2012). Drug-loaded CSs demonstrate sustained release profiles, which may explain the promotion of drug's retention in skin (Tan et al., 2011; Hu et al., 2014). These published data are consistent with our results. In this study, we indeed verified that Qu-loaded TPP-Chitosan NPs (QTCs) could enhance the skin permeation of Qu by changing the morphology of the stratum corneum and enhance the skin retention of $\mathrm{Qu}$.

It is worth mentioning that CS has inherent antibacterial and antimycotic properties due to its polycationic nature, which can inhibit the growth of a variety of pathogenic microorganisms Gram-positive and Gram-negative bacteria, yeasts and other fungi (Rabea et al., 2003; Ignatova et al., 2013). Furthermore, CSs could also inhibit the growth of various bacteria, including E. coli, S. choleraesuis, S. typhimurium, and S. aureus (Qi et al., 2004).

Owing to these advantages, CSs can be an ideal carrier for topical drug and gene delivery (Özbas̨-Turan and Akbuğa, 2011; Yang et al., 2013). Our study firstly elucidates the advantage of using Qu-loaded CSs for topical use to prevent UVB radiationinduced skin damage. As displayed in Figure 6, our finding showed that the UVB radiation stimulates the phosphorylation of IkB- $\alpha$ and subsequent degradation of IkB- $\alpha-N F-\kappa B$ complex, which makes the activated NF- $\kappa$ B translocate to the nucleus from the cytoplasm. Nucleus NF- $\kappa \mathrm{B}$ then activates the expression of COX-2, the key enzyme converting AA into prostaglandins (PGE2). Excess PGE2 increases the vascular permeability and promotes edema and further damage. Qu is well-known for inhibiting NF- $\kappa$ B/COX-2 pathway, but its topical use is largely restricted by its natural disadvantages of low hydrophilicity

\section{REFERENCES}

Ali, S. M., and Yosipovitch, G. (2013). Skin pH: from basic science to basic skin care. Acta Derm. Venereol. 93, 261-267. doi: 10.2340/00015555-1531

de Alencar Filho, J. M. T., Sampaio, P. A., Valenccedil, E. C., Pereira, A., Gonccedil, R., Juacute, A. D. O., et al. (2016). Flavonoids as photoprotective agents: a systematic review. J. Med. Plants Res. 10, 848-864. doi: 10.5897/ JMPR2016.6273

DiDonato, J. A., Hayakawa, M., Rothwarf, D. M., Zandi, E., and Karin, M. (1997).

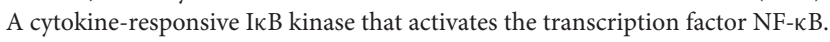
Nature 388, 548-554. doi: 10.1038/41493

Eberleinkönig, B., Schäfer, T., Hussmarp, J., Darsow, U., Möhrenschlager, M., Abeck, D., et al. (2000). Skin surface pH, stratum corneum hydration, transepidermal water loss and skin roughness related to atopic eczema and skin dryness in a population of primary school children. Acta Derm. Venereol. 80, 188-191. doi: 10.1080/000155500750042943

Enríquez, D. S. A., Diebold, Y., Calonge, M., García-Vazquez, C., Callejo, S., Vila, A., et al. (2006). Chitosan nanoparticles as a potential drug delivery system for the ocular surface: toxicity, uptake mechanism and in vivo tolerance. Invest. Ophthalmol. Vis. Sci. 47, 1416-1425. doi: 10.1167/iovs.050495 and poor percutaneous absorption. However, in QTCs drug delivery system, TCs contributes to overcome the natural disadvantages of $\mathrm{Qu}$, resulting in better hydrophilicity and low cytotoxicity of QTCs drug delivery system, and also help to significantly increase the percutaneous absorption, retention and cellular uptake of $\mathrm{Qu}$ in the skin, which further enhances the inhibition of the NF- $\mathrm{B} / \mathrm{COX}-2$ signaling pathway and prevents skin from UVB radiation-induced damage.

\section{CONCLUSION}

Our present study firstly demonstrates that the Qu-loaded chitosan NPs have the ability to prevent the UVB radiationinduce skin damage and can be used as the promising therapeutic agent.

\section{AUTHOR CONTRIBUTIONS}

XjS and WN conceived the idea and designed the study. WN and LD performed all the experiments, analyzed the data, and co-wrote the paper. HC and LY provided technical support. FK helped correcting the manuscript. $\mathrm{XjS}$ and $\mathrm{XbS}$ provided reagents and conceptual advice.

\section{FUNDING}

This work was supported by grants from National Natural Science Foundation of China (Grant No. 81730108), Key Project of Zhejiang province Ministry of Science and Technology (Grant No. 2015C03055), Key Project of Hangzhou Ministry of Science and Technology (Grant Nos. 20162013A07 and 20142013A63), and Shenzhen Science and Technology Program (Grant Nos. JCYJ20170412153453623 and JCYJ20130402145002433).

Formica, J. V., and Regelson, W. (1995). Review of the biology of Quercetin and related bioflavonoids. Food Chem. Toxicol. 33, 1061-1080. doi: 10.1016/02786915(95)00077-1

Gallagher, R. P., and Lee, T. K. (2006). Adverse effects of ultraviolet radiation: a brief review. Prog. Biophys. Mol. Biol. 92, 119-131. doi: 10.1016/j.pbiomolbio. 2006.02.011

Hatahet, T., Morille, M., Hommoss, A., Devoisselle, J. M., Müller, R. H., and Bégu, S. (2016). Quercetin topical application, from conventional dosage forms to nanodosage forms. Eur. J. Pharm. Biopharm. 108, 41-53. doi: 10.1016/j.ejpb. 2016.08.011

Hu, X., Wang, Y., and Peng, B. (2014). Chitosan-capped mesoporous silica nanoparticles as $\mathrm{pH}$-responsive nanocarriers for controlled drug release. Chem. Asian J. 9, 319-327. doi: 10.1002/asia.201301105

Huang, M., Ma, Z., Khor, E., and Lim, L. Y. (2002). Uptake of FITC-chitosan nanoparticles by A549 cells. Pharm. Res. 19, 1488-1494. doi: 10.1023/A: 1020404615898

Hung, C. F., Fang, C. L., Alsuwayeh, S. A., Yang, S. Y., and Fang, J. Y. (2012). Evaluation of drug and sunscreen permeation via skin irradiated with UVA and UVB: comparisons of normal skin and chronologically aged skin. J. Dermatol. Sci. 68, 135-148. doi: 10.1016/j.jdermsci.2012. 09.005 
Hur, S., Lee, Y. H., Yang, J. H., and Kim, T. Y. (2010). Homoisoflavanone inhibits UVB-induced skin inflammation through reduced cyclooxygenase-2 expression and NF-kappaB nuclear localization. J. Dermatol. Sci. 59, 163-169. doi: 10.1016/j.jdermsci.2010.07.001

Ignatova, M., Manolova, N., and Rashkov, I. (2013). Electrospun antibacterial chitosan-based fibers. Macromol. Biosci. 13, 860-872. doi: 10.1002/mabi. 201300058

Jiao, J., Mikulec, C., Ishikawa, T. O., Magyar, C., Dumlao, D. S., Dennis, E. A., et al. (2014). Cell-type-specific roles for COX-2 in UVB-induced skin cancer. Carcinogenesis 35, 1310-1319. doi: 10.1093/carcin/bgu020

Kerr, J. B., and Mcelroy, C. T. (1993). Evidence for large upward trends of ultraviolet-b radiation linked to ozone depletion. Science 262, 1032-1034. doi: $10.1126 /$ science.262.5136.1032

Kim, S. O., Kundu, J. K., Shin, Y. K., Park, J. H., Cho, M. H., Kim, T. Y., et al. (2005). [6]-Gingerol inhibits COX-2 expression by blocking the activation of p38 MAP kinase and NF-kappaB in phorbol ester-stimulated mouse skin. Oncogene 24, 2558-2567. doi: 10.1038/sj.onc. 1208446

Liu, X. J., Shi, S. T., Ye, J. L., Liu, L. Q., Sun, M., and Wang, C. B. (2009). Effect of polypeptide from Chlamys farreri on UVB-induced ROS/NF-kappaB/COX2 activation and apoptosis in HaCaT cells. J. Photochem. Photobiol. B Biol. 96, 109-116. doi: 10.1016/j.jphotobiol.2009.04.010

Madronich, S., and Gruijl, F. R. D. (1993). Skin cancer and UV radiation. Nature 366:23. doi: $10.1038 / 366023 \mathrm{a} 0$

Mccarty, M. F. (2012). Minimizing the cancer-promotional activity of cox-2 as a central strategy in cancer prevention. Med. Hypotheses 78, 45-57. doi: 10.1016/ j.mehy.2011.09.039

Morganroth, P. A., Lim, H. W., and Burnett, C. T. (2013). Ultraviolet radiation and the skin: an in-depth review. Am. J. Lifestyle Med. 7, 168-181. doi: 10.1177/ 1559827612460499

Özbaş-Turan, S., and Akbuğa, J. (2011). Plasmid DNA-loaded chitosan/TPP nanoparticles for topical gene delivery. Drug Deliv. 18, 215-222. doi: 10.3109/ 10717544.2010 .544688

Pal, H. C., Athar, M., Elmets, C. A., and Afaq, F. (2015). Fisetin inhibits UVBinduced cutaneous inflammation and activation of PI3K/AKT/NFKB signaling pathways in SKH-1 hairless mice. Photochem. Photobiol. 91, 225-234. doi: 10. 1111/php. 12337

Pentland, A. P., Schoggins, J. W., Scott, G. A., Khan, K. N., and Han, R. (1999). Reduction of UV-induced skin tumors in hairless mice by selective COX-2 inhibition. Carcinogenesis 20, 1939-1944. doi: 10.1093/carcin/20.10.1939

Qi, L., Xu, Z., Jiang, X., Hu, C., and Zou, X. (2004). Preparation and antibacterial activity of chitosan nanoparticles. Carbohydr. Res. 339, 2693-2700. doi: 10. 1016/j.carres.2004.09.007

Rabea, E. I., Badawy, E. T., Stevens, C. V., Smagghe, G., and Steurbaut, W. (2003). Chitosan as antimicrobial agent: applications and mode of action. Biomacromolecules 4, 1457-1465. doi: 10.1021/bm034130m

Schipper, N. G., Olsson, S., Hoogstraate, J. A., Deboer, A. G., Vårum, K. M., and Artursson, P. (1997). Chitosans as absorption enhancers for poorly absorbable drugs 2: mechanism of absorption enhancement. Pharm. Res. 14, 923-929. doi: 10.1023/A:1012160102740
Seda Tığlı Aydın, R., and Pulat, M. (2012). 5-Fluorouracil encapsulated chitosan nanoparticles for $\mathrm{pH}$-stimulated drug delivery: evaluation of controlled release kinetics. J. Nanomater. 2012:313961. doi: 10.1155/2012/313961

Serafini, M., Peluso, I., and Raguzzini, A. (2010). Flavonoids as anti-inflammatory agents. Proc. Nutr. Soc. 69, 273-278. doi: 10.1017/S002966511000162X

Steerenberg, P. A., Garssen, J., Dortant, P. M., Vliet, H. V. D., Geerse, E., Verlaan, A. P. J., et al. (1997). The effect of oral quercetin on UVB-induced tumor growth and local immunosuppression in SKH-1. Cancer Lett. 114, 187-189. doi: 10.1016/S0304-3835(97)04659-4

Tan, Q., Liu, W., Guo, C., and Zhai, G. (2011). Preparation and evaluation of quercetin-loaded lecithin-chitosan nanoparticles for topical delivery. Int. J. Nanomed. 6, 1621-1630. doi: 10.2147/IJN.S22411

Tang, S. C., Liao, P. Y., Hung, S. J., Ge, J. S., Chen, S. M., Lai, J. C., et al. (2017). Topical application of glycolic acid suppresses the UVB induced IL-6, IL-8, MCP-1 and COX-2 inflammation by modulating NF- $\mathrm{B}$ signaling pathway in keratinocytes and mice skin. J. Dermatol. Sci. 86, 238-248. doi: 10.1016/j. jdermsci.2017.03.004

Tang, X., Kim, A. L., Kopelovich, L., Bickers, D. R., and Athar, M. (2008). Cyclooxygenase-2 inhibitor nimesulide blocks ultraviolet B-induced photocarcinogenesis in SKH-1 hairless mice. Photochem. Photobiol. 84, 522-527. doi: 10.1111/j.1751-1097.2008.00303.x

Yang, R., Shim, W. S., Cui, F. D., Cheng, G., Han, X., Jin, Q. R., et al. (2009). Enhanced electrostatic interaction between chitosan-modified PLGA nanoparticle and tumor. Int. J. Pharm. 371, 142-147. doi: 10.1016/j.ijpharm. 2008.12.007

Yang, Y., Bugno, J., and Hong, S. (2013). Nanoscale polymeric penetration enhancers in topical drug delivery. Polym. Chem. 4, 2651-2657. doi: 10.1016/ j.xphs.2016.08.022

Zhang, Y., Yang, Y., Tang, K., Hu, X., and Zou, G. (2008). Physicochemical characterization and antioxidant activity of quercetin-loaded chitosan nanoparticles. J. Appl. Polym. Sci. 107, 891-897. doi: 10.1002/app.26402

Zhu, X., Zeng, X., Zhang, X., Cao, W., Wang, Y., Chen, H., et al. (2016). The effects of quercetin-loaded PLGA-TPGS nanoparticles on ultraviolet B-induced skin damages in vivo. Nanomedicine 12, 623-632. doi: 10.1016/j.nano.2015.10.016

Conflict of Interest Statement: LY was employed by company Shenzhen Modo Biotech Co., Ltd.

The remaining authors declare that the research was conducted in the absence of any commercial or financial relationships that could be construed as a potential conflict of interest.

Copyright (c) 2018 Nan, Ding, Chen, Khan, Yu, Sui and Shi. This is an open-access article distributed under the terms of the Creative Commons Attribution License (CC BY). The use, distribution or reproduction in other forums is permitted, provided the original author(s) and the copyright owner(s) are credited and that the original publication in this journal is cited, in accordance with accepted academic practice. No use, distribution or reproduction is permitted which does not comply with these terms. 